\title{
NUMERICAL SIMULATION AND EXPERIMENTAL VALIDATION OF A HYPERSONIC FLOW FOR NUMERICAL MODULATION OF RE-ENTRY PHENOMENA PREDICTION USING ADAPTIVE MESH REFINEMENT
}

\author{
S. REICHEL \& R. GROLL \\ Center of Applied Space Technology and Microgravity, University of Bremen, Bremen, Germany.
}

\begin{abstract}
The subject of interest is the validation of a 3-D numerical computer model of a hypersonic flow around double cone geometry. The double cone geometry represents a generic space vehicle which enters the atmosphere at extremely high velocity. This leads to complex flow phenomena around the space vehicle. In this paper the flow-field around the space vehicle is investigated.

Experimental data is obtained for different double-cone geometries mounted inside a hypersonic wind-tunnel. During the experiments the Mach number is equal to 9. Three different geometries and four different operating conditions are the subject of this study. Because of the short test period of less than $200 \mathrm{~ms}$ a measurement of temperatures and local velocities is not possible during test. Therefore, the computational model is used.

The numerical solver is based on the compressible Navier-Stokes equations and implements an adaptive meshing tool. This solver is used for flow-field simulations of re-entry phenomena. The cell refinement tool adapts the local cell length to the density gradient. In this way, all shock-waves receive higher resolution than the remaining mesh and the solver shows good agreement with the experimental results while minimizing computational cost and time. For this purpose of this study a basic open source solver is used and modified and solutions are validated on experimental data.

The aim of this paper is to show a good agreement of experimental pressure measurements and numerical results and to estimate results of the temperature field, the velocity field and the local Mach number using the numerical model.
\end{abstract}

Keywords: CFD, compressible flow, hypersonic, Navier-Stokes equations, OpenFOAM, re-entry, supersonic.

\section{INTRODUCTION}

An important issue for the design of spacecraft vehicles is the effect of various flow phenomena around the spacecraft, especially for atmospheric entry. Due to the fact that the velocity of a spacecraft is highly hypersonic during re-entry, understanding these flow phenomena is essential for the design of a spacecraft. Therefore, a lot of academic and fundamental research has been done in this field. Different studies analyze the flow phenomena around spacecrafts by using numerical methods (e.g. [1-3]). Other authors investigated unsteady flow phenomena of different nose geometries in experiments and computer simulations (e.g. [4, 5]). These authors mostly used 2-D computer-simulations because of the high complexity of their simulations, and because 3-D meshes would overcharge their computer systems. For this reason the authors also use symmetric boundary conditions.

The aim of this paper is the validation of a 3-D numerical computational fluid dynamics model with experimental results. The understanding of the flow phenomena, especially the local temperatures, velocities, with Mach numbers and the position and strength of the shock waves are also of interest. To this end, several different generic double-cone geometries are placed inside a hypersonic Mach 9 wind-tunnel. During the experiments the pressure along the double-cones are measured. These measurements are compared to simulation results. 
The presented numerical results are calculated on a 3-D mesh. Because of the complexity of 3-D results, the numerical solver utilizes an adaptive meshing tool. This tool enables the increase and decrease the local cell-length. In this way, the solver offers the possibility to refine and coarsen the mesh in according to the solver settings. Thus, the flow phenomena around the investigated regions are resolved more finely than the remaining mesh. Areas of high refinement are those areas with high density fluctuations, in general the boundary layer between shock-wave and double-cone. In this way computational cost is severely reduced and the numerical 3-D problem becomes manageable on a common system. An equally resolved static mesh would overcharge common systems.

In this paper the following approach is made. First the governing equations of the numerical solver are presented. Then the benefit of using adaptive mesh refinement is presented. It is shown that the modified solver reaches the same final solution as the static mesh solver while minimizing system resources and time. Then the new solver is used to resolve the complex flow phenomena around double-cone geometries in 3-D. The results are compared to measurement data and discussed. A good agreement between measurement and numerical results is presented and discussed.

\section{COMPUTATIONAL METHODS AND GOVERNING EQUATIONS}

The simulations are conducted with a modified OpenFOAM solver 'rhoCentralFoam'. This solver solves the continuity eqn (1), the Navier-Stokes equations eqn (2) and energy eqn (3). The detailed equations are presented below. The general derivations of these three equations are given in [6].

$$
\begin{gathered}
\frac{\partial \rho}{\partial t}+\nabla \cdot(\rho \vec{u})=0 \\
\rho\left[\frac{\partial \vec{u}}{\partial t}+\vec{u} \cdot \nabla \vec{u}\right]=-\nabla p-\nabla \cdot \tau \\
\frac{\partial \rho E}{\partial t}+\nabla[\vec{u}(\rho E)]=-\nabla \cdot[p \vec{u}]-\nabla \cdot[\tau \cdot \vec{u}]-\nabla \cdot \vec{j}
\end{gathered}
$$

The continuity eqn (1) defines that the change of density $\partial \rho / \partial t$ inside the system is equal to the net flux of mass $\nabla \cdot(\rho \vec{u})$ over the system boundaries. The Navier-Stokes Equation eqn (2) consist of the unsteady acceleration $\partial \vec{u} / \partial t$, of the convective acceleration $(\vec{u} \cdot \nabla \vec{u})$, of the pressure gradient $(\nabla p)$, and the divergence of the viscous stress tensor $\tau$. $I$ is the identity matrix tensor.

$$
\tau=-2 \mu\left(D-\frac{1}{3} \operatorname{tr}(D) I\right)
$$

The energy eqn (3) includes the total energy density

$$
E=e+\frac{|\vec{u}|^{2}}{2}
$$

and the divergence of the diffusive flux of heat $\vec{j}$ which is proportional to temperature gradient $\nabla T$ and the thermal conductivity coefficient $k$ :

$$
\vec{j}=-k \nabla T
$$


Standard pressure correction methods often show bad agreement between computational results and experimental data for compressible hypersonic flows. Therefore, a different method is used by implementing own numerical procedures. The solver includes an explicit predictor equation and an implicit corrector equation for the diffusion of primitive variables instead of a pressure correction. The procedure is the well-known Kurganov's method (e.g. $[7,8])$. This reference solver was programmed by Greenshields and Welter. In general, the Kurganov flux correction corrects the pressure field and the diffusive flux fields, which means a correction of the diffusive terms of the momentum equation and the energy equation. Thus the fluxes of $\rho, \phi, \psi$, and $e$ are interpolated at cell surfaces. The fluxes are averaged inside the cell volume in combination with a weighting procedure and used in the iteration process of the solver. A detailed description of this solver is given in [9].

\section{ADAPTIVE MESH REFINEMENT}

An additional feature of the modified OpenFOAM solver is the adaptive mesh refinement. This tool is able to refine local cells, depending on the density gradient $(\nabla \rho)$. The threshold of the refinement tool defines which cells are refined. To activate the mesh refinement tool some essential changes to the solver's source-code are necessary. After the modifications, the mesh refinement tool searches for cells with high density gradients which exceed a userdefined value. The located cells are selected for the cell refinement. Therefore, the selected cells are divided into 8 sub-cells. This means that the cell borders are split into two parts in all three dimensions $\left(2^{3}=8\right.$ cells $)$. This leads to a new locally refined mesh. All calculated results of the basic cell are transferred to the new mesh and the iteration process is continued. The cell refinement level is user-defined which means every cell is divisible in n-sub-cells. Only one refinement level per time-step is possible.

The refinement level is limited by the basic mesh. Only previously refined cells which fall below the user-defined critical value are coarsened to the next larger cell size.

To speed up the iteration process, it is practical not to refine cells in every time-step. The presented results of this paper are calculated on a mesh which was modified every 100 timesteps. In this way, the dynamics of the process is well resolved and the final solution is reached in an acceptable time. To make sure that the new adaptive mesh solver leads to the same final solution, a benchmark test is performed on the same computer system. A comparison of final results for pressure, temperature, velocity and density permits evaluation of the solution quality of the adaptive mesh solver. The time taken to reach the final solution is also of interest.

Therefore, a basic low resolved mesh consisting of 16128 cells is constructed. This mesh is used for a simulation with the static solver and the adaptive solver. A third simulation was performed on a completely refined mesh consisting of 1032192 cells. In this way the cell lengths of the refined basic mesh is equal to the cell lengths of the completely refined mesh.

The performed test-case describes a supersonic flow over a forward facing step. The boundary conditions of all three test cases are identical.

The plots in Figs 1-3 show a comparison of the three different test cases. The first picture shows the result of the statically refined mesh and the basic mesh, the second picture the result of the static high resolved mesh and the last picture shows the result of the adaptive mesh solver. The pictures are colored by pressure field. The result of the statically refined mesh and the adaptive mesh solver are the same. The positions of pressure gradients (shock waves) are identical. The first result is different to the other results. It can be seen that the flow shows the same basic characteristics as the other two simulations, but the shock waves are at different positions especially at the near-wall region. This is caused by the lower local resolution. 


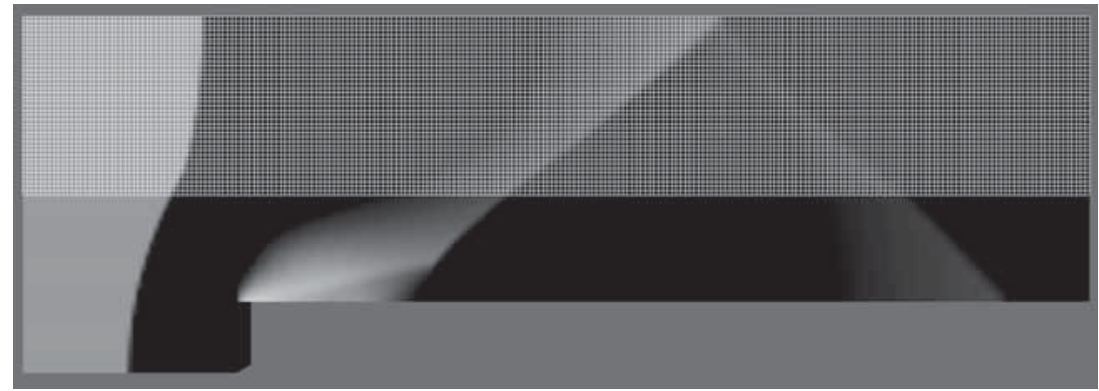

Figure 1: Final result of pressure field using low resolved static mesh.

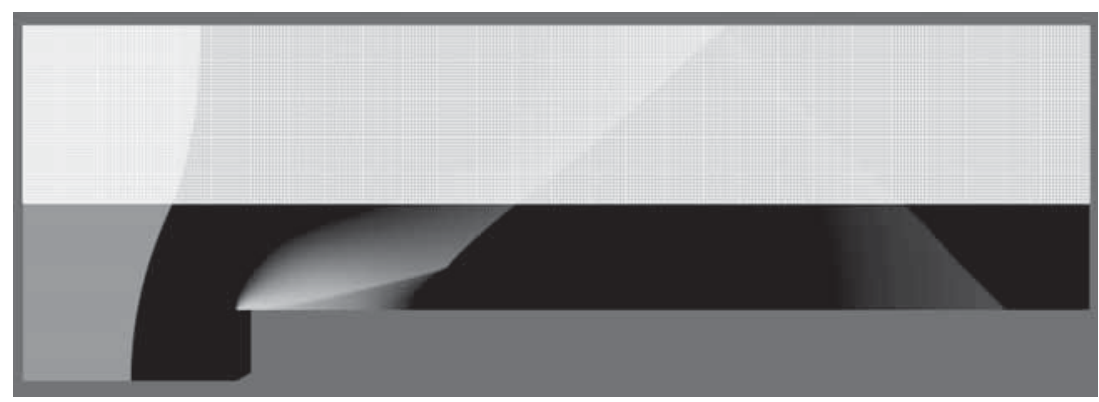

Figure 2: Final result of pressure field using high resolved static mesh.

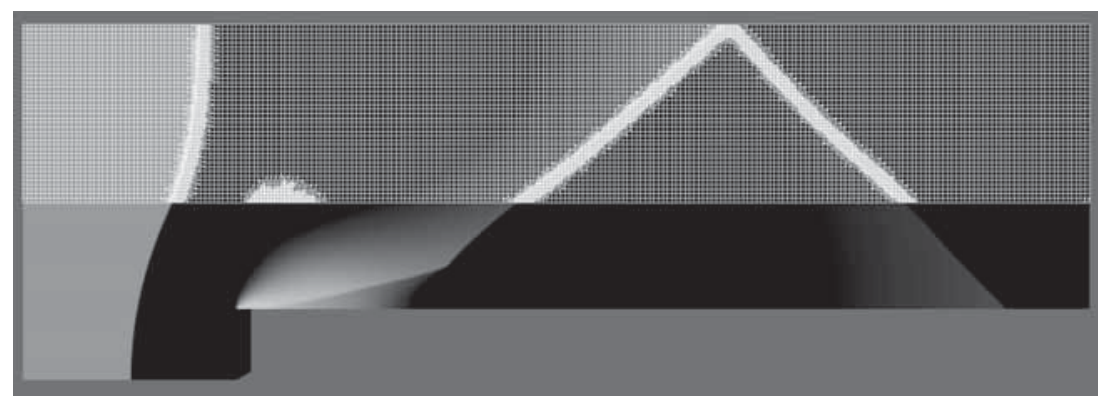

Figure 3: Final result of pressure field using adaptive mesh refinement.

Figure 4 compares the final result of pressure, density, temperature and velocity fields of all three simulations at the horizontal center line of the simulation region. It is seen that the deviations of the final solution of the completely refined mesh and the dynamic mesh are minute. These two results are in good agreement. The results of the basic low resolved mesh are not the same. The shock waves are at different positions. The main difference between the adaptive mesh simulation and the completely resolved mesh simulation is the computational cost. The adaptive mesh simulation takes 110000 s. The static mesh simulation takes about 450000s. This means that the adaptive meshing solver is more than four times faster than the static mesh solver for the presented benchmark test. This speedup is 

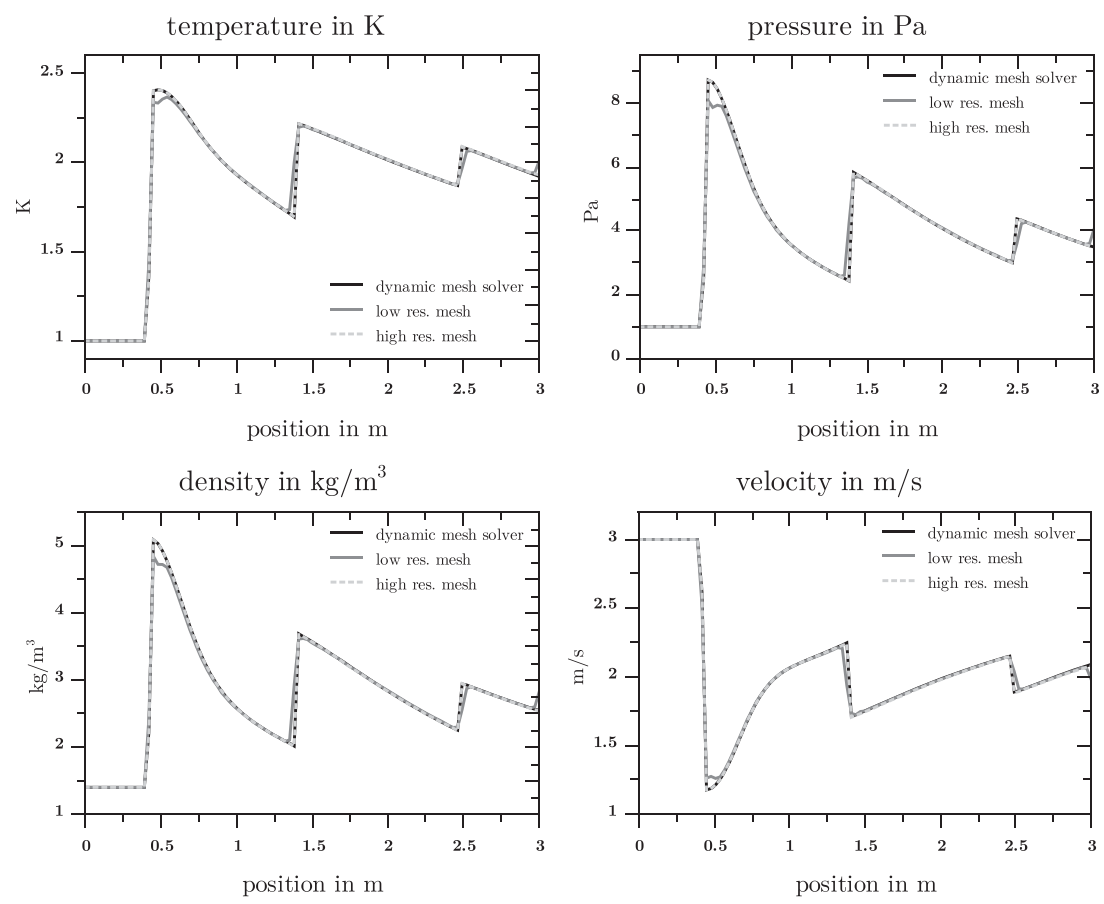

Figure 4: Comparison of the numerical results of the forward step simulations of the basic low resolved mesh in combination with the static and the adaptive solver and the result of the completely resolved mesh in combination with the static solver.

caused by the smaller mesh. The adaptive mesh solver uses a minimum of cells. This means the mesh is smaller than 180000 cells. The static mesh solver uses a mesh with more than 1000000 cells. In this way the adaptive mesh solver saves a lot of computational cost and it is possible to reinvest this sources in a more detailed or complex simulation. Figure 5 shows the transient flow solution of the adaptive mesh refinement solver of the forward step simulation at various times. These pictures are colored by pressure field. The basic mesh is refined at the black areas.

\section{EXPERIMENTAL SETUP OF THE TEST FACILITY}

The experimental set up is mounted inside the 'ZARM hypersonic wind-tunnel'. The wind-tunnel facility is a special kind of pressure vacuum tunnel called Ludwig tube. The large evacuated chamber is separated by a fast acting valve. At the upstream end of the valve a nozzle is connected to a long cylindrical tube. The air pressure and temperature inside this tube are adjustable.

Figure 6 shows the Bremer Hochschul Hyperschallkanal BHHK (University of Bremen hypersonic wind tunnel). Inside the charging tube air is heated up to $900 \mathrm{~K}$ and pressurized up to $10 \mathrm{MPa}$ (Fig. 6). To start the process the valve opens rapidly and a shock wave propagates into the low-pressure region. In this way the hypersonic wind-tunnel generates a constant hypersonic speed in a range of $6 \leq M a \leq 11$ for $200 \mathrm{~ms}$.

Figure 7 shows the different double-cone geometries which are used for the validation test of the numerical solver. The major difference between these three conical geometries is 


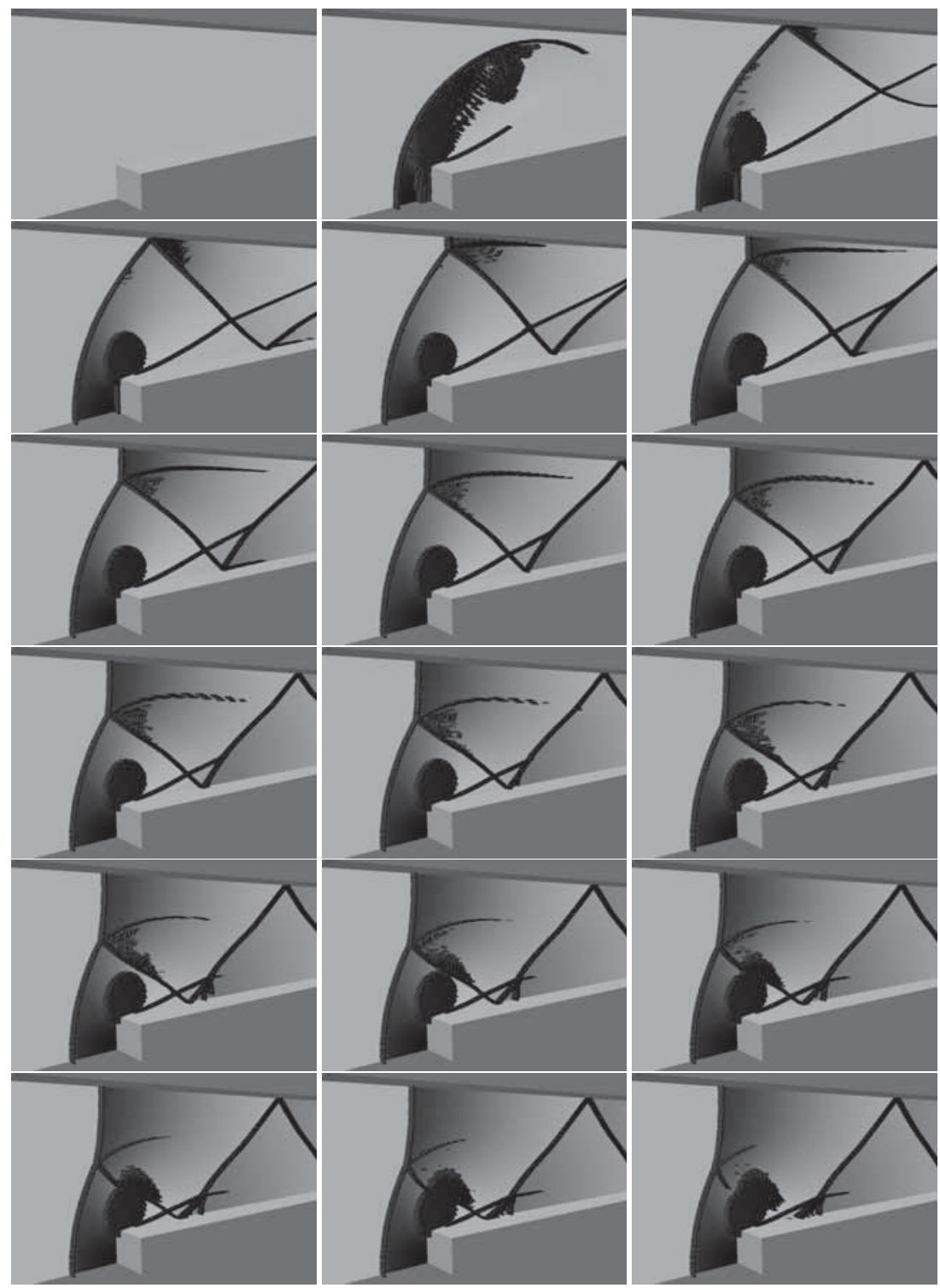

Figure 5: Transient flow solution at various times for the forward facing step simulation. The basic mesh is refined at the black areas.

their tip. The first double-cone has a spherical head with a diameter of $20 \mathrm{~mm}$, the second cone a spherical head with a diameter of $10 \mathrm{~mm}$ and the third cone has a sharp cone end. The small cavities along the double-cones are the spots of pressure measurement during operation time.

The notation $\mathrm{R}(A)-(B)(C)$ of the different double-cone geometries results from the radius $(A)$ of curvature at the peak of the geometry in millimeters, the angle $(B)$ of the first cone and 


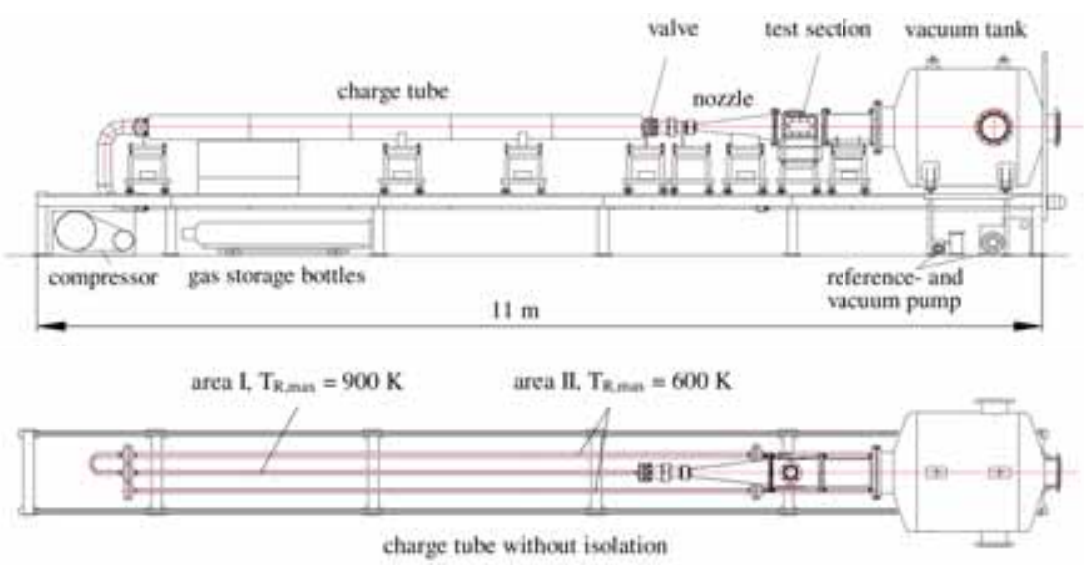

Figure 6: Geometrical wind-tunnel set-up: side-view (top) and top-view (bottom).

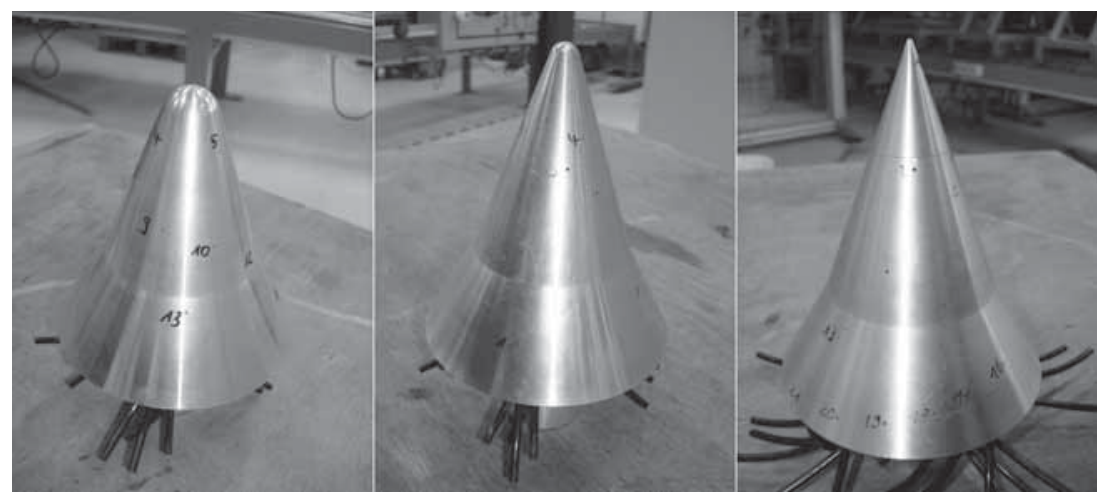

Figure 7: Double cone geometries R10-2030 (left), R05-2030 (middle) and R00-2030 (right).

the angle $(C)$ of the second cone in degrees. The different geometries are shown in Fig. 8. Therefore, the 'nose' of the R10-2030 geometry has a larger radius of curvature than the R05-2030 geometry.

\section{BOUNDARY CONDITIONS}

To define the boundary conditions for the numerical solution some basic definitions according to [10] are used. For isotropic and ideal gas the following equations of the total temperature eqn (7), the Mach number eqn (8), and the pressure eqn (9) are defined.

The following values are known for the experimental setup during operation time. The Mach number is equal to 9, the temperature inside the air accumulator tube of the windtunnel is $T_{t}=900 \mathrm{~K}$, the isentropic exponent of air is estimated as $k=1.4$. This means that the temperature effect on the Mach number of the isotropic exponent is ignored for the boundary condition estimation. Reason for this decision is the variation of temperature inside the test facility. During experiments the temperature varied in a range of $50 \mathrm{~K}$ up to $900 \mathrm{~K}$. Therefore, eqn (7) is used. 


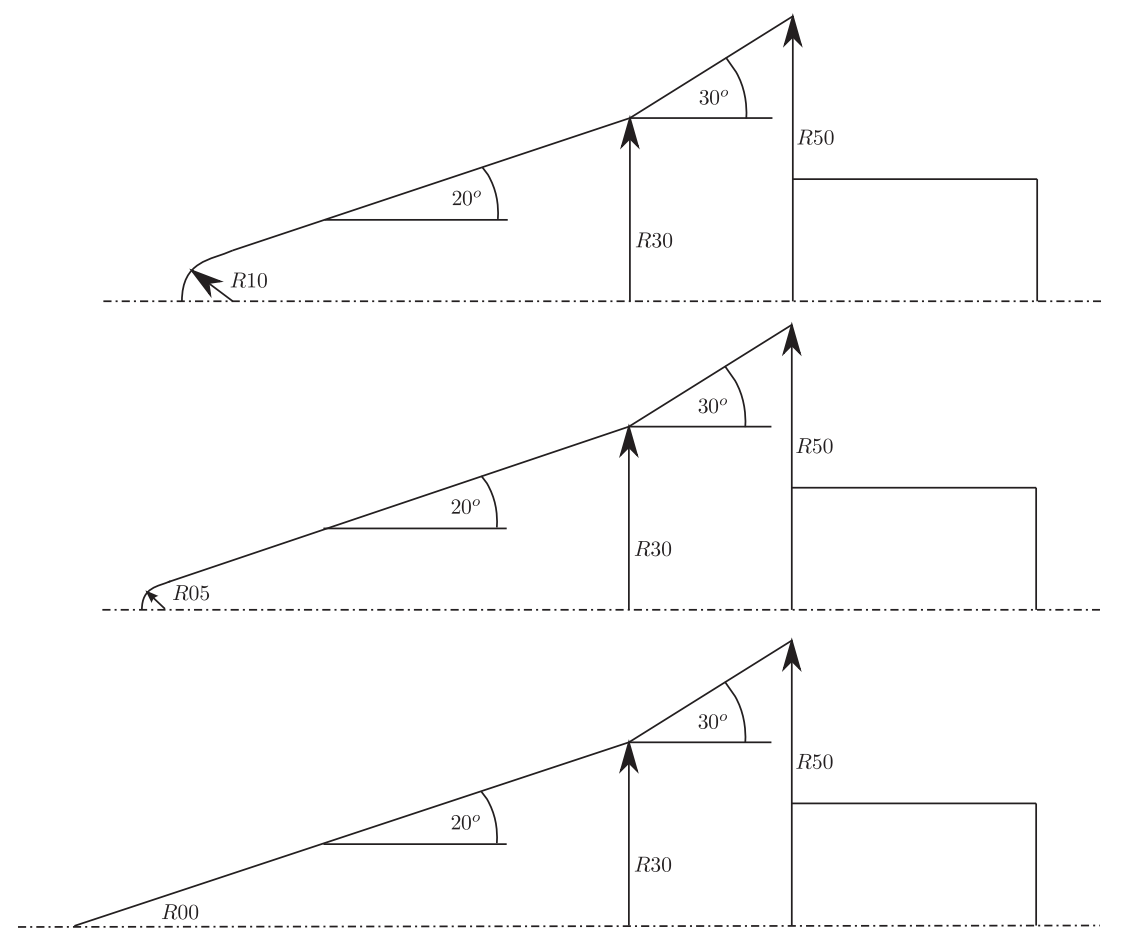

Figure 8: Shape of the three different cone geometries.

$$
\frac{T_{m}}{T_{t}}=\left(1+\frac{k-1}{2} \cdot M a^{2}\right)-1=\frac{5}{86}
$$

leads to a temperature of the initial flow field of $T_{m} \approx 52.326 \mathrm{~K}$. With the molar mass of $M=0.02896 \frac{\mathrm{kg}}{\mathrm{mol}}$, the universal gas constant of $R=8.3145 \frac{\mathrm{J}}{\mathrm{mol} \mathrm{K}}$ and using eqn (8)

$$
c=\sqrt{k \cdot \frac{R T}{M}}
$$

the speed of sound is $c \approx 145.02 \frac{\mathrm{m}}{\mathrm{s}}$. With a Mach number equal 9 the free stream velocity is $u=1305.2 \frac{\mathrm{m}}{\mathrm{s}}$.

The pressure in the air accumulator tube is in the range of $4 \mathrm{MPa}<p_{t}<10 \mathrm{MPa}$. In this case the relation presented in eqn (9)

$$
\frac{p_{m}}{p_{t}}=\left[\frac{T_{m}}{T_{t}}\right]^{\frac{k}{k-1}} \approx 4.739 \cdot 10^{-5}
$$

defines the free-stream pressure inside the test-length within a range of $189.5 \mathrm{~Pa} \leq p_{m}$ $\leq 473.9 \mathrm{~Pa}$. The measured values are between $183 \mathrm{~Pa} \leq p_{m} \leq 463 \mathrm{~Pa}$. This depends on the pressure inside the air accumulator tube of the wind-tunnel and negligible pressure losses. 
The following boundary conditions are used for the presented simulation results of the $p_{t}=10 M P a$ cases:

- Inlet: Basics of previous solver experiences shows that boundary conditions which are controlled by a ramp function leads to higher numerical stability of the solver. For this propose the boundary condition the velocity is controlled by a ramp function which increases the initial velocity at the inlet from $U_{\text {initial }}=200 \frac{\mathrm{m}}{\mathrm{s}}$ up to $U_{\text {final }}=1302 \frac{\mathrm{m}}{\mathrm{s}}$ during the first $4 \mathrm{~ms}$ of simulation time. The temperature is fixed at $T=52 \mathrm{~K}$. The pressure is fixed at $p=463 \mathrm{~Pa}$.

- Outlet: The outlet boundary conditions for temperature, pressure and velocity are defined as 'zeroGradient'. This means all these variables are calculated from the results of the interior domain.

- Wall: The wall boundary conditions are used for the surface of the double-cone geometry. The velocity is set to $U=0 \frac{\mathrm{m}}{\mathrm{s}}$. This leads to a no-slip boundary condition for the doublecone geometry. The heat fluxes at the wall are neglected during the simulation time. The heat flux is very low, because of the short test period of $100 \mathrm{~ms}$. Therefore, the wall is modeled adiabatically.

- Internal Field: This boundary condition defines the initial values of temperature, pressure and velocity of the internal field. The values are set to $T=52 \mathrm{~K}, \mathrm{p}=463 \mathrm{~Pa}$ and $U_{\text {Field }}=100 \frac{\mathrm{m}}{\mathrm{s}}$.

To calculate the other operating conditions of the experiments only the system pressure must be changed.

Because of the used estimations of the boundary conditions of the numerical simulations, postprocessing was used for system check. The Mach number has very strong effect on the system flow field especially at the pressure destruction. Therefore, postprocessing is used for the calculation of the Mach number. The postprocessing shows a flow velocity of $M a=$ 9.00613. This means a deviation of the Mach number is $1 \%$ larger than demanded.

\section{NUMERICAL MOCK-UP}

The basic geometry was meshed with a 3-D meshing tool and is similar to the test cones of the experiment. The only difference between the experimental geometry and the numerical geometry is at the downstream region of the double-cones. Because of a hypersonic flow, the sharp angle behind the double-cone geometries has no influence on the upstream flow-field. To reduce the calculation time the downstream area of all used double-cone geometries are simplified in this area.

The basic meshes consist of about 700,000 hexahedral cells. The double-cone geometries shown in Fig. 8 are subjected to this study.

\section{MEASUREMENT DATA AND COMPUTATIONAL RESULTS}

The plots in Fig. 9 compare the experimental measurements (black dots and error bars) and the numerical data (gray lines and error-bars). The measuring rate of the pressure sensors equals $1000 \mathrm{~Hz}$. For the evaluation of the experimental data the results are averaged over ten measured values. The standard deviation of the experimental data is calculated and presented. The gray line is the averaged surface-tangential value of the simulation. The shown standard 

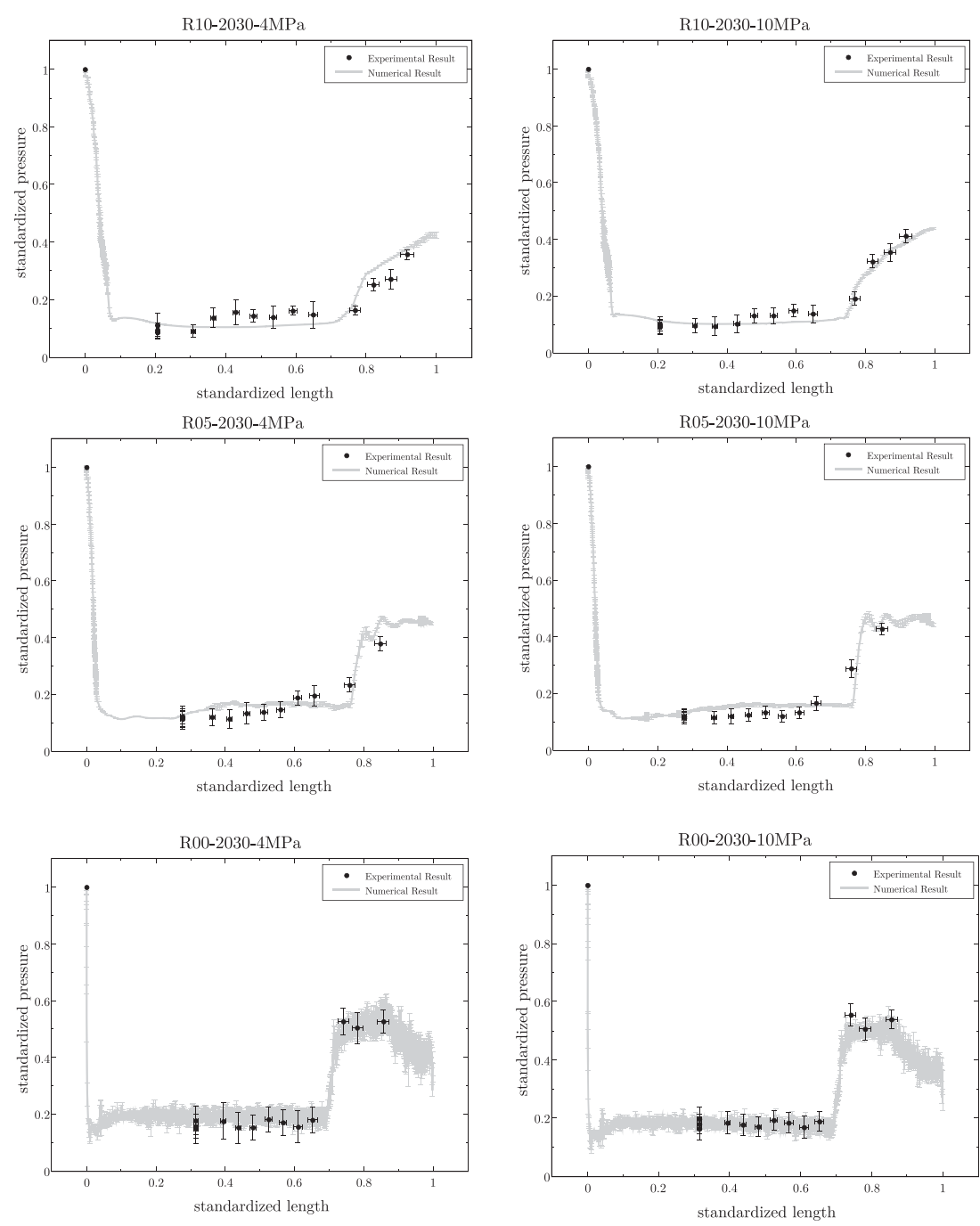

Figure 9: Surface pressure distribution of the experiments for all three double-cone geometries at different charging pressure of $4 M P a$ and $10 M P a$ of the charging tube.

deviation is calculated from the variation of these surface-tangential values. The y-axes of these plots shows the normalized pressure and the x-axes show the normalized length of the double-cone geometry.

The pressure is normalized with the respective static pressure at the cone tip. The length is normalized by the geometry. Therefore, the position of the cone tip (the position of the respective dynamic pressure) is equal to 0 and the end position of the edge of the related double cone is equal to 1 .

Three different double-cone geometries and four different operating conditions are simulated and tested. For the generation of the four different test conditions the pressure inside the air accumulator tube of the wind-tunnel is varied from $4 M P a$ up to $10 M P a$. Higher pressure inside the air accumulator tube leads to higher mass-flow through the system and this leads 
to higher pressures at the surface of the double-cone geometry during the experiments. This relation also conforms to eqns (7)-(9).

The normalized results of measurement and simulation data are very similar. The comparison of all test series of the respective double-cones shows that the pressure values behave in the same manner. The deviations between the four different operating conditions of the measurement and the simulation results are within the related standard deviation although there are small geometric deviations between the experimental test cones and the numerical mesh. The experimental test cones have small cavities for the pressure measurement along the cone geometry as is shown in Fig. 7. These cavities have been ignored in the generation of the CFD mesh. Figure 10 shows the simulation result of surface pressure distribution and the flow fields around the R10-2030 double-cone.

The value of the standard deviation of the pressure is a degree of unsteady flow-field along the double-cone geometry. As is visible in the plots of Fig. 9, the shape of the nose has a heavy influence on the pressure variabilities in the near-wall area. The reason for this phenomenon is the geometry of the related cone. A ball headed cone like the R10-2030 and R05-2030 generates a less unsteady flow than the sharp edge headed R00-2030 cone. The phenomenon of pressure variabilities is visible in the experimental and the numerical results in the same manner. These phenomena of unsteady flow-field around hypersonic test objects are subjects of many other studies (e.g. [5, 11]).

Also of interest is the temperature along the double cone geometry. Figure 11 shows that the shape also has a strong influence on the local temperatures. The highest temperatures are reached at the tip of the cone. For all simulations the maximum temperatures are all of the same order. The temperature of R00-2030 reaches $T_{N}=919 K$, the R05-2030 reaches $T_{N}=969 \mathrm{~K}$ and the R10-2030 reaches $T_{N}=915 \mathrm{~K}$. The temperature deviations at the tip of the double cone geometry for the different operation conditions are smaller than $1 \%$.

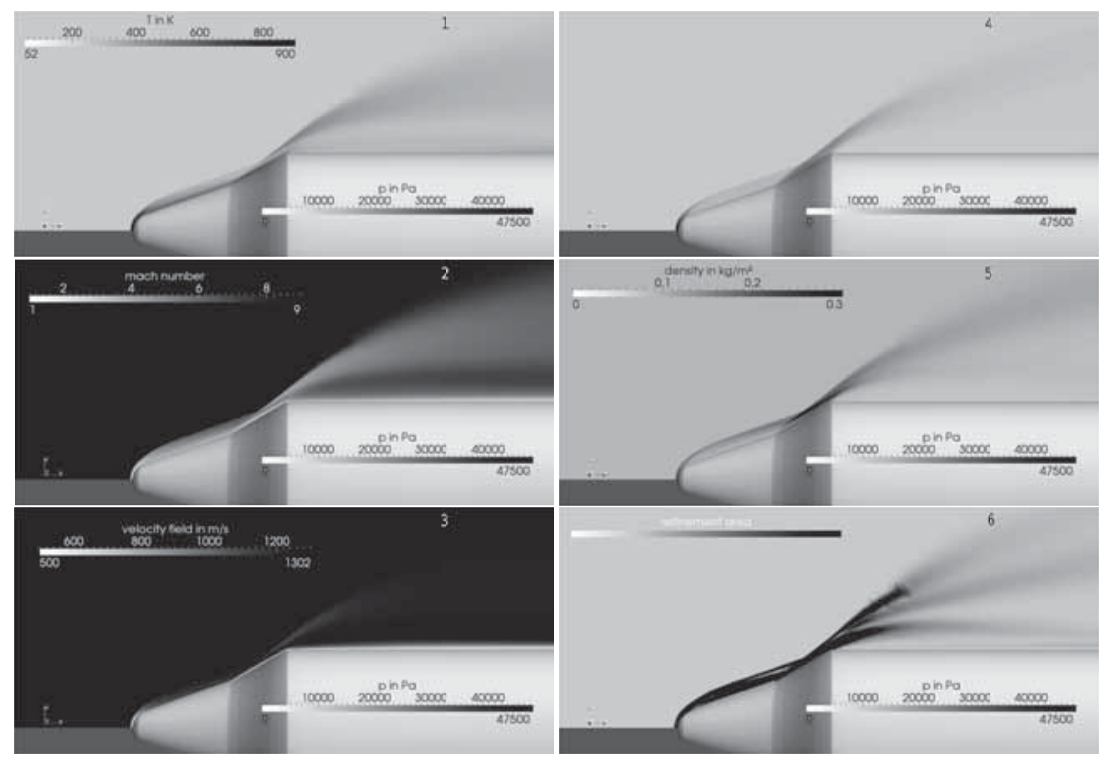

Figure 10: Final result of the R10-2030 double-cone simulation. First picture shows tempererature, second local Mach number, third velocity, fourth pressure, fifth density field and the sixth the refinement area (black colored). 

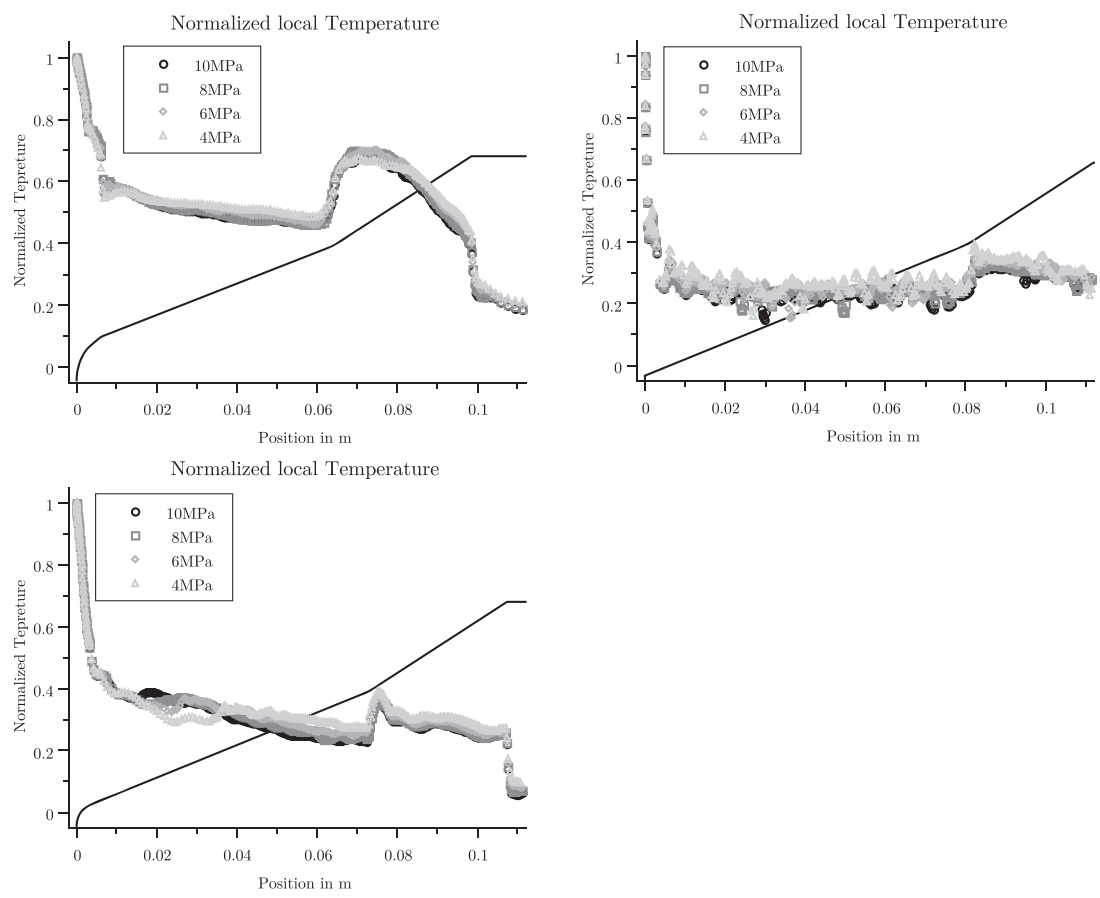

Figure 11: Surface temperature distribution along the different double-cone geometries for all tested operation conditions (normalized with $T_{N}$ ).

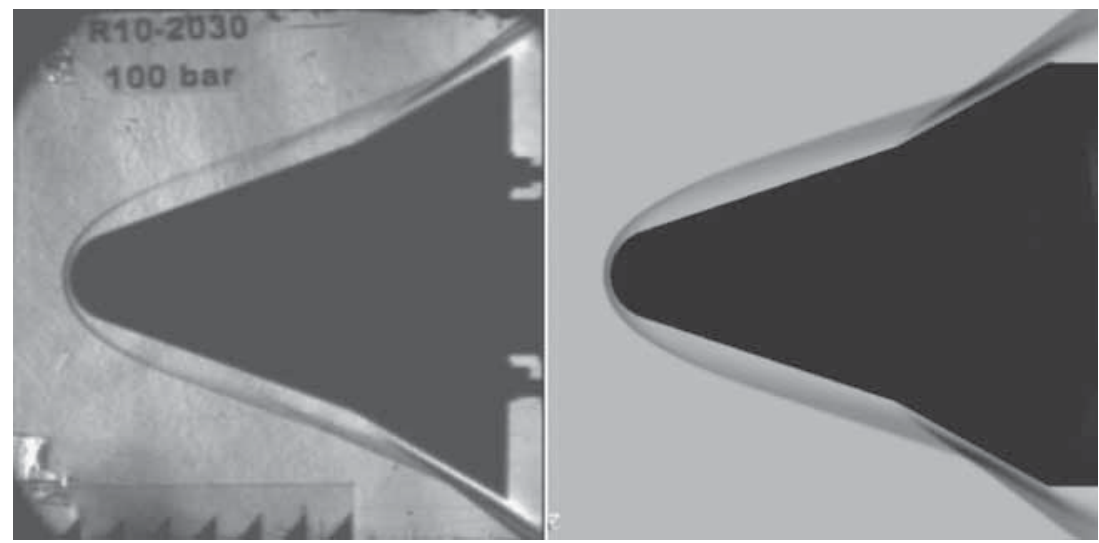

Figure 12: Left-hand side photography of a striation optic during operation (experimental result). Right-hand side final solution colored by density (numerical result).

The temperature is independent of the mass-flow through the system. The plots in Fig. 11 shows the normalized temperature distribution of the different operation conditions.

Figure 12 shows an image of the working experiment (left-hand side) and the solution of the numerical solver (right-hand side) for the R10-2030 double cone. The picture on the left-hand side is taken with a striation optic, the picture on the right-hand side is extracted 
from the numerical solution and colored by the total density. These two pictures are in good agreement. The deviations are very low and it is conceivable that these deviations arise from two different sources. The first source is the color scaling of the numerical data. The relation between black level and density of the striation optic is not known. So the coloration of the right-hand side picture has to be estimated. The second source of deviation is a different view. The experimental image illustrates a 3-D experiment. This means it contains density information of all three dimensions. The right-hand side illustrates a 2-D cut through the numerical solution. This image contains only information about two dimensions. Although there is some difference between these two pictures, the comparison shows that the density increase and decrease are practically at the same position.

\section{CONCLUSIONS}

Three different double-cones have been tested in a hypersonic wind-tunnel at a $M a=9$. The results of the experiments were used for a validation test on a numerical solver. The operating mode of the numerical model was illustrated. The used mesh and the used boundary conditions are displayed and explained. The solver implements an adaptive meshing tool which increases the local number of cells in dependence on the local density gradient. This leads to a complete resolved mesh around the double-cone geometry, especially in the region between the shock-wave and the test object.

For all presented numerical test-cases the adaptive mesh refinement tool works correctly and stable and the region between the shock-wave and the double-cone geometry is higher resolved than the basic mesh. The results of the experiments and the numerical solution are presented and related in normalized plots. These plots show good agreement between the experiments and the numerical model for all three double-cones and the different operating conditions.

Finally, a photograph of a striation optic during operation and a rendering of the final computational solution are compared to each other. Both pictures display the same behavior of the flow-field around the double-cone.

Further investigations are underway to implement additional terms into the source code of the numerical solver for the description of additional forces which act on the flow-field. The next stage of solver development is to implement electric heating inside the flow-field due to an electric ignition between an anode and a cathode.

\section{ACKNOWLEGEMENTS}

The authors thank Holger Oelze and Tibo Helms for performing the experiments and their contribution to this work. Additionally many thanks are directed at Ronald Mairose for preparation of the test facility and Kristofer Leach for his support.

\section{NOMENCLATURE}

$$
\begin{aligned}
& \rho=\text { mass density } \\
& \phi=\text { mass flux }(\mathrm{v} \cdot p) \\
& \psi=\text { specific heat } \\
& v=\text { velocity } \\
& \mu=\text { dynamic viscosity coefficient } \\
& p=\text { pressure } \\
& p_{0}=\text { local pressure } \\
& \nabla=\text { Nabla Operator } \\
& \nabla^{2}=\text { Laplace Operator } \\
& \partial t=\text { time step }
\end{aligned}
$$


$e \quad=$ specific internal energy

$\mathrm{E}=$ total energy density

$I \quad=$ tensor of unity

$D=$ deformation gradient tensor $D$

$T=$ viscous stress tensor $\tau$

$j \quad=$ diffusive flux of heat $\vec{j}$

$k=$ thermal conductivity coefficient

$T_{m}=$ temperature of the flow-field

$T_{t}=$ temperature inside the air accumulator tube

$c=$ speed of sound

$\kappa=$ isentropic exponent

$M a=$ Mach number

$M=$ molar mass

$R=$ universal gas constant

\section{REFERENCES}

[1] Xu, K., Mao, M. \& Tang, L., A multidimensional gas-kinetic BGK scheme for hypersonic viscous flow. Journal of Computational Physics, 203, pp. 405-421, 2005. doi: http://dx.doi.org/10.1016/j.jcp.2004.09.001

[2] Candler, G.V., Nompelis, I., Druguet, M.-C., Holden, M.S., Wadhams, T.P., Boyd, I.D. \& Wang, W.-L., CFD validation for hypersonic flight: hypersonic double-cone flow simulations. AIAA Paper, No. 2002-0581, 2002.

[3] Bucchignani, E. \& Pezzella, G., Computational flowfield analyses of hypersonic problems with reacting boundary layer. Mathematics and Computers in Simulation, No. MATCOM-3362, 2010.

[4] Gauer, M. \& Paull, A., Numerical investigation of a spike blunt nose cone at hypersonic speeds. Journal of Spacecraft and Rockets, 45(3), pp. 459-471, 2008, doi: http://dx.doi. org/10.2514/1.30590.

[5] Viren Menezes, Saravanan, S., Jagadeesh, G. \& Reddy, K.P.J., Experimental investigations of hypersonic flow over highly blunted cones with Aerospikes. AIAA Journal, 41(10), pp. 1955-1966, 2003. doi: http://dx.doi.org/10.2514/2.1885

[6] Oertel, H., Jr., Böhle, M. \& Dohrmann, U., Strömungsmechanik, 5th edn., Vieweg+Teubner I GWV Fachverlage GmbH, Wiesbaden, 2009, Chaps. 3, 2.

[7] Kurganov, A. \& Tadmor, E., New high-resolution central schemes for nonlinear conservation laws and convection-diffusion equations. Journal of Computational Physics, 160, pp. 241-282, 2000. doi: http://dx.doi.org/10.1006/jcph.2000.6459

[8] Kurganov, A., Noelle, S. \& Petrova, G., Semidiscrete central-upwind schemes for hyperbolic conservation laws and Hamilton-Jacobi equations. Society for Industrial and Applied Mathematics, 23(3), pp. 707-740, 2001.

[9] Greenshields, C.J., Weller, H.G., Gasparini, L. \& Reese, J.M., Implementation of semidiscrete, non-staggered central schemes in a colocated, polyhedral, finite volume framework, for high-speed viscous flows. International Journal for Numerical Methods in Fluids, 63, pp. 1-23, 2009. doi: http://dx.doi.org/10.1002/fld.2069

[10] Ferziger, J.H. \& Perić, M., Computational Methods for Fluiddynaimcs, 2nd edn., Springer Verlag, Berlin, 1997, Chaps. 10, 2.

[11] Furumoto, G.H., Zhong, X. \& Skiba, J.C., Numerical studies of real-gas effects on two-dimensional hypersonic shock-wave/boundary-layer interaction. Physics of Fluids, 9, pp. 191-211, 1997. doi: http://dx.doi.org/10.1063/1.869162 